University of Wollongong

Research Online

Faculty of Social Sciences - Papers (Archive) Faculty of Arts, Social Sciences \& Humanities

$1-1-2018$

\title{
Can explicit suggestions about the harmfulness of EMF exposure exacerbate a nocebo response in healthy controls?
}

Adam Verrender

University of Wollongong, av138@uowmail.edu.au

Sarah P. Loughran

University of Wollongong, loughran@uow.edu.au

Anna Dalecki

University of Wollongong, adalecki@uow.edu.au

Frederik Freudenstein

University of Wollongong, frederik@uow.edu.au

Rodney J. Croft

University of Wollongong, rcroft@uow.edu.au

Follow this and additional works at: https://ro.uow.edu.au/sspapers

Research Online is the open access institutional repository for the University of Wollongong. For further information contact the UOW Library: research-pubs@uow.edu.au 


\title{
Can explicit suggestions about the harmfulness of EMF exposure exacerbate a nocebo response in healthy controls?
}

\author{
Abstract \\ 2018 Elsevier Inc. While there has been consistent evidence that symptoms reported by individuals who \\ suffer from Idiopathic Environmental Intolerance attributed to Electromagnetic Fields (IEI-EMF) are not \\ caused by EMF and are more closely associated with a nocebo effect, whether this response is specific to \\ IEI-EMF sufferers and what triggers it, remains unclear. The present experiment tested whether perceived \\ EMF exposure could elicit symptoms in healthy participants, and whether viewing an 'alarmist' video \\ could exacerbate a nocebo response. Participants were randomly assigned to watch either an alarmist ( $\mathrm{N}$ \\ $=22)$ or control video $(\mathrm{N}=22)$ before completing a series of sham and active radiofrequency (RF) EMF \\ exposure provocation trials ( 2 open-label, followed by 12 randomized, double-blind, counterbalanced \\ trials). Pre- and post-video state anxiety and risk perception, as well as belief of exposure and symptom \\ ratings during the open-label and double-blind provocation trials, were assessed. Symptoms were higher \\ in the open-label RF-ON than RF-OFF trial $(p<.001)$. No difference in either symptoms $(p=.183)$ or belief of \\ exposure $(p=.144)$ was observed in the double-blind trials. Participants who viewed the alarmist video \\ had a significant increase in symptoms $(p=.041)$, state anxiety $(p<.01)$ and risk perception $(p<.001)$ \\ relative to the control group. These results reveal the crucial role of awareness and belief in the \\ presentation of symptoms during perceived exposure to EMF, showing that healthy participants exhibit a \\ nocebo response, and that alarmist media reports emphasizing adverse effects of EMF also contribute to \\ a nocebo response.

\section{Publication Details} \\ Verrender, A., Loughran, S., Dalecki, A., Freudenstein, F. \& Croft, R. (2018). Can explicit suggestions about \\ the harmfulness of EMF exposure exacerbate a nocebo response in healthy controls?. Environmental \\ Research, 166 409-417.
}




\section{Can explicit suggestions about the harmfulness of EMF exposure exacerbate a nocebo response in healthy controls?}

Adam Verrender ${ }^{\text {a, b* }}$, Sarah P. Loughran ${ }^{\text {a, b, c }}$, Anna Dalecki ${ }^{\text {b, c }}$, Frederik Freudenstein ${ }^{\text {a, b, c }}$, Rodney J. Croft ${ }^{\text {a, b, c }}$

${ }^{\text {a }}$ Australian Centre for Electromagnetic Bioeffects Research

${ }^{\mathrm{b}}$ School of Psychology, Illawarra Health \& Medical Research Institute, University of Wollongong, Wollongong, Australia

${ }^{\mathrm{c}}$ Population Health Research on Electromagnetic Energy, Monash University, Melbourne, Australia

Adam Verrender: av138@uowmail.edu.au～Bachelor of Psychology (Honours)

Sarah P. Loughran: loughran@uow.edu.au_ Doctor of Philosophy

Anna Dalecki: $\quad$ adalecki@uow.edu.au_ Doctor of Philosophy

Frederik Freudenstein: frederik@uow.edu.au_ Doctor of Philosophy

Rodney J. Croft: $\quad$ rcroft@uow.edu.au_ Doctor of Philosophy

*Corresponding author: Adam Verrender. Australian Centre for Electromagnetic Bioeffects Research, Illawarra Health and Medical Research Institute, Bld 32.113, University of Wollongong, Northfields Ave, Wollongong, NSW, 2522, Australia Tel: +612 4239 2118; Fax: +612 4221 8130; Email: av138@uowmail.edu.au 


\begin{abstract}
While there has been consistent evidence that symptoms reported by individuals who suffer from Idiopathic Environmental Intolerance attributed to Electromagnetic Fields (IEI-EMF) are not caused by EMF and are more closely associated with a nocebo effect, whether this response is specific to IEI-EMF sufferers and what triggers it, remains unclear. The present experiment tested whether perceived EMF exposure could elicit symptoms in healthy participants, and whether viewing an 'alarmist' video could exacerbate a nocebo response. Participants were randomly assigned to watch either an alarmist $(N=22)$ or control video $(N$ = 22) before completing a series of sham and active radiofrequency (RF) EMF exposure provocation trials (2 open-label, followed by 12 randomized, double-blind, counterbalanced trials). Pre- and post-video state anxiety and risk perception, as well as belief of exposure and symptom ratings during the open-label and double-blind provocation trials, were assessed. Symptoms were higher in the open-label RF-ON than RF-OFF trial $(p<.001)$. No difference in either symptoms $(p=.183)$ or belief of exposure $(p=.144)$ was observed in the doubleblind trials. Participants who viewed the alarmist video had a significant increase in symptoms $(p=.041)$, state anxiety $(p<.01)$ and risk perception $(p<.001)$ relative to the control group. These results reveal the crucial role of awareness and belief in the presentation of symptoms during perceived exposure to EMF, showing that healthy participants exhibit a nocebo response, and that alarmist media reports emphasizing adverse effects of EMF also contribute to a nocebo response.
\end{abstract}

\title{
Keywords:
}

Electromagnetic hypersensitivity; Idiopathic environmental intolerance attributed to electromagnetic fields (IEI-EMF); Media reports; Medically unexplained symptoms 
Funding: This work was supported by the National Health and Medical Research Council of Australia [grant numbers: 1042464, 2012; 1060205, 2013] and the Australian Government Research Training Program Scholarship. The funding source had no involvement in any aspects of the study design, data collection, analysis and interpretation of findings, writing of the paper or decision to submit the paper for publication.

Competing Interest Statement: The authors have no competing interests to report.

Ethics: The study was approved by the University of Wollongong Human Research Ethics Committee (HE: 2016/981). 


\section{Introduction}

The public's perception of the potential health implications associated with the use of modern technologies has been steadily changing in recent years (Petrie et al., 2001; Petrie \& Wessely, 2002). This is often reflected in the mainstream media, where news reports consistently suggest that there are dangers of various aspects of modern life while often neglecting more mundane causes of illness (Frost, Frank, \& Maibach, 1997; Petrie \& Wessely, 2002). Generally, these stories do not reflect the current state of science (Claassen, Smid, Woudenberg, \& Timmermans, 2012; Eldridge-Thomas \& Rubin, 2013), but instead focus on reports of members of the community who claim to experience conditions characterized by a variety of adverse symptoms which they ascribe to their use of, or proximity to, various environmental stimuli, including vaccinations, genetically modified food, infrasound from wind turbines and electromagnetic fields (EMF) emitted by mobile phone and wireless technologies (Petrie \& Wessely, 2002).

One particularly prominent condition is Idiopathic Environmental Intolerance attributed to Electromagnetic Fields (IEI-EMF). People who suffer from this condition typically report experiencing a diverse range of non-specific symptoms which they attribute to their exposure to the EMF emitted by everyday electrical and wireless technologies and infrastructure (Baliatsas, Van Kamp, Lebret, \& Rubin, 2012; Röösli, Moser, Baldinini, Meier, \& BraunFahrländer, 2004). Yet, while a considerable proportion of the population report experiencing IEI-EMF (estimated to be between 1.5 - 13.5\% (Baliatsas et al., 2015; Blettner et al., 2009; Eltiti, Wallace, Zougkou, et al., 2007; Hillert, Berglind, Arnetz, \& Bellander, 2002; Levallois, Neutra, Lee, \& Hristova, 2002; Schreier, Huss, \& Röösli, 2006; Schröttner \& Leitgeb, 2008; Tseng, Lin, \& Cheng, 2011)), there has been no robust evidence to implicate a bioelectromagnetic mechanism in producing the reported symptoms (Health Canada, 2015; Health Council of the Netherlands, 2009; Röösli, Frei, Mohler, \& Hug, 2010; Rubin, Das 
Munshi, \& Wessely, 2005; Rubin, Nieto-Hernandez, \& Wessely, 2010; Scientific Committee on Emerging and Newly Idenified Health Risks, 2015; Staudenmayer, Binkley, Leznoff, \& Phillips, 2003). For instance, when tested under double-blind protocols, IEI-EMF participants do not report an increase in symptoms to EMF and are unable to perceive the difference between active and sham exposures (Röösli et al., 2010; Rubin et al., 2010). Instead, the evidence suggests that the condition is more closely associated with a nocebo response, as awareness of the exposure and a belief of being exposed have been shown to play an important role in the presentation of the condition. For example, a number of studies have found that participants experience an increase in symptoms when they are aware of the active exposure condition in an initial non-blinded trial compared to sham, but do not exhibit more symptoms in active than sham exposures in subsequent double-blind trials (Eltiti, Wallace, Ridgewell, et al., 2007; van Moorselaar et al., 2017; Verrender et al., 2018). Furthermore, sham exposures (ie. with no EMF) have been shown to be sufficient to trigger symptoms in IEI-EMF participants (Nam et al., 2009; Oftedal, Straume, Johnsson, \& Stovner, 2007; Verrender et al., 2018; Wilén, Johansson, Sandström, Kalezic, \& Lyskov, 2006). The exact role of the nocebo response in the development of IEI-EMF, however, is not fully understood. For instance, recent findings from a qualitative study suggest that instead of the condition originating from a nocebo response, IEI-EMF individuals may be using the notion of sensitivity to EMF to provide a narrative to explain their pre-existing medically unexplained symptoms, in an effort to make their condition more practically and emotionally manageable Dieudonné (2016). Yet, it is important to note that Dieudonné (2016) did not test the cause of the participants symptoms, but rather, retrospectively asked participants about their beliefs regarding the cause of their symptoms. As retrospective self-reports are known to suffer from recall bias (Baliatsas et al., 2015; Vrijheid et al., 2009), these methods are not able to determine symptom etiology. 
Given the prevalence of distressing and debilitating IEI-EMF symptoms, and in light of the evidence suggesting that such symptoms may be the result of a nocebo response, there is a great need to better understand the triggers that elicit such responses. Generally, a nocebo response occurs when conscious or subconscious negative expectations trigger or exacerbate adverse symptoms in response to an exposure that is not known to cause those effects (Bräscher, Kleinböhl, Hölzl, \& Becker, 2017; Hahn, 1997). These expectations may be induced by explicit suggestions about the potential effects of an exposure (Benedetti, Lanotte, Lopiano, \& Colloca, 2007; Webster, Weinman, \& Rubin, 2016) or by learning through classical conditioning (Bräscher, Kleinböhl, et al., 2017).

The communication of information about potential adverse health effects associated with EMF exposure constitutes an explicit suggestion which may be responsible for the formation of negative expectations and consequent nocebo response seen in IEI-EMF individuals (Webster et al., 2016). For example, there has been consistent evidence that precautionary information can negatively influence beliefs about EMF exposure, despite this information originally being intended to reassure the public (Barnett, Timotijevic, Shepherd, \& Senior, 2007; Nielsen et al., 2010; Wiedemann, Boerner, \& Repacholi, 2014; Wiedemann et al., 2013; Wiedemann \& Schütz, 2005; Wiedemann, Thalmann, Grutsch, \& Schütz, 2006). Similarly, viewing mainstream media reports which either promote the view that EMF exposure is hazardous, or focus on individuals with IEI-EMF, have been shown to increase worries about EMF exposure (Witthöft et al., 2017), while viewing an advertisement claiming to protect against the 'harmful effects of everyday EMF exposure' has been shown to increase both heart rate and concern about EMF (Köteles, Tarján, \& Berkes, 2016). Further, recent content analyses have shown that mainstream media reports about EMF exposure often misrepresent the current state of scientific evidence by focusing on an electromagnetic cause for IEI-EMF, or suggesting a relationship between EMF exposure and 
ill-health (Claassen et al., 2012; Eldridge-Thomas \& Rubin, 2013). If such misinformation is being distributed on a wide scale and is negatively influencing people's beliefs about EMF exposure, it is possible that this may be a contributing factor to the prevalence of IEI-EMF.

Yet, it remains unclear whether the negative beliefs induced by such communications can result in greater symptom formation following a perceived exposure to EMF. Although Szemerszky, Köteles, Lihi, and Bárdos (2010) demonstrated that suggestions about the strength of EMF exposure can lead to increased symptom scores and an increase in the belief that a sham magnetic field was active, that study did not assess the effect of explicit suggestions of risk from EMF exposure (which may induce negative expectations) and was limited by a lack of counterbalancing. Furthermore, while Witthöft and Rubin (2013) reported that viewing a sensationalist media report about the adverse effects of Wi-Fi can increase the likelihood of a person experiencing symptoms following a sham exposure and developing an apparent sensitivity to EMF, the effect was only found for those with high preexisting levels of state anxiety. This may be because the study lacked a verified non-exposure condition, potentially resulting in insufficient statistical power to detect effects in nonanxious individuals. In support of this notion, a similar study which included a cued nonexposure condition found that those who watched a film focusing on 'adverse effects of WiFi’ perceived tactile electrical stimuli as more intense during a cued Wi-Fi exposure (which was actually a sham exposure) compared to a cued no Wi-Fi condition, and that the effect was not mediated by anxiety (Bräscher, Raymaekers, Van den Bergh, \& Witthöft, 2017). This suggests that manipulating a participant's belief of exposure via cues may be important for influencing symptom perception irrespective of pre-existing state anxiety levels. The latter study, however, assessed somatosensory perception rather than symptom perception, and so it remains uncertain as to whether negative beliefs induced by information about EMF exposure 
can result in greater symptom formation or belief regarding exposure status following a perceived exposure to EMF.

To address these limitations, the present study was designed to determine whether perceived EMF exposure could elicit symptoms in a healthy population, and additionally, whether messages emphasizing 'adverse health effects of EMF exposure' can exacerbate a nocebo response. The study was also designed to explore, within-subjects, whether there is a relationship between a person's belief of exposure and symptoms, and whether there is a difference in symptom response between participants with low, medium and high pre-existing levels of state anxiety. To this end, an initial non-blinded open-label trial was employed, where the status of exposures emanating from the device (during an active and sham condition) were visually demonstrated to each participant using an EMF meter.

\section{Materials and Methods}

\subsection{Participants}

Forty-four participants aged 18 - 30 years $(M=21.92, S D=4.88 ; 50 \%$ male) were recruited through advertisements placed online and around the University of Wollongong campus. A power calculation conducted in G*Power 3.0 (Faul, Erdfelder, \& Buchner, 2007) for an independent samples $t$ test based on an effect size of 0.8 , an alpha level of .05 and a power of 0.80 recommended a total sample size of 42 .

All participants were first screened via a telephone interview to confirm eligibility for the study. To be included in the study, participants were required to be over the age of 18 and report being of good health. Participants were excluded from the study if they reported having a current illness or medical condition, or having used illicit substances within the 7day period prior to the study. Suitable participants were required to attend the Illawarra Health and Medical Research Institute for one mutually convenient testing session. The study 
was approved by the Human Research Ethics Committee (HE: 2016/981). All participants were instructed to abstain from alcohol for at least $8 \mathrm{~h}$, caffeine for at least $1 \mathrm{~h}$, and mobile phone use for at least $2 \mathrm{~h}$ before the beginning of the testing session. Participants were compensated with a monetary gift card for their involvement in the study.

\subsection{Radiofrequency exposure}

RF exposure was generated using a portable, self-contained, battery-operated device (Two Fields Consulting, St Kilda, Australia). The RF device was placed $30 \mathrm{~cm}$ to the left side of the participant (at approximately shoulder to head height) on a hard surface. The main exposure from the device was a spread spectrum RF signal in the 902-928 MHz ISM band which was digitally modulated in a similar manner to signals from Wi-Fi and 3G/4G mobile phones. The RF signal was generated by a commercial RF modem which emitted a frequency hopping spread spectrum signal with an average radiated power output of $1 \mathrm{~W}$ for $10 \mathrm{~min}$ (RF-ON), or was completely EMF silent (RF-OFF, sham trials). The incident RF exposure level from the side of the device facing the participant was measured using a calibrated broadband instrument with an uncertainty of $\pm 2.4 \mathrm{~dB}$ for a two-sided coverage interval and a coverage factor of 2 (Narda EMR 300 meter and Type 9 E-field probe, Narda Safety Test Solutions, Hauppauge, NY), and was found to be $0.3 \mathrm{~W} / \mathrm{m}^{2}$. This RF exposure level is below the power density reference level limit of $4.6 \mathrm{~W} / \mathrm{m}^{2}$ specified for the Australian general public (ARPANSA RPS3) and by the International Commission on Non-Ionizing Radiation Protection (ICNIRP, 1998). It is important to note that the maximum localized specific absorption rate (SAR) from the exposure device used in the present study is less than that induced from personal mobile phone use (held against the ear in the active talk mode) due to the greater separation distance. Conversely, the whole body averaged SAR and localized SAR of the device are greater than those normally produced by Wi-Fi and mobile phone base station signals. The device was fully enclosed in a thermally insulated case and coded inputs 
were used to maintain double-blinding. The device contained an independent RF monitor to check the status of the RF transmitter and each use of the device was logged using internal memory.

\subsection{Questionnaires}

\subsubsection{Symptoms and exposure status scale (SESS)}

During the provocation trials, participants were asked to indicate whether they believed the exposure was on or off, and to rate whether they were experiencing any symptoms via pen and paper $100 \mathrm{~mm}$ visual analogue scales. To assess belief of exposure, participants were asked "how sure are you of the current exposure status right now?" anchored with the terms 'Definitely OFF' and 'Definitely ON'. To assess symptom experience, a modified state version of the 34 item Checklist for Symptoms in Daily Life (Wientjes \& Grossman, 1994; Witthöft \& Rubin, 2013) was used. Participants were asked "how strong/unpleasant are the following symptoms right now?” anchored with the terms 'Barely Detectable' and 'Maximum Severity'. These response categories differed from the original questionnaire (Wientjes \& Grossman, 1994) and were used in line with our previous study (Verrender et al., 2018). The symptom responses of the 34 items were added to calculate a total symptom score for each of the baseline and exposure intervals in each trial. The primary dependent variables for belief of exposure and symptoms in the provocation trials were calculated as difference scores between the baseline and exposure questionnaires (exposure interval minus preceding baseline) given during each trial (see procedure below); a difference score was used to minimize the influence of baseline variability and potential carry-over effects.

\subsubsection{Risk perception questionnaire (RPQ)}

A self-generated risk perception questionnaire comprising 4 questions was used to assess EMF risk perception. Question 1 assessed concerns about electromagnetic fields in general 
and question 2 assessed concerns about electromagnetic fields in relation to mobile phones and Wi-Fi. Participants were asked "How concerned are you about the potential health risks of electromagnetic fields in general?” rated on a 7-point Likert scale $(1=$ not worried at all, 7 = very worried) and “All in all, how threatened do you feel by electromagnetic radiation emissions from mobile phones and Wi-Fi?” rated on a 7-point Likert scale $(1=$ not threatening at all, 7 = very threatening). To enable standardized measurement of RF-EMF risk perception in relation to mobile phones and $\mathrm{Wi}-\mathrm{Fi}$, questions 3 and 4 used picture-guided scenarios which illustrated everyday exposure situations (Freudenstein, Wiedemann, \& Brown, 2015). Participants were asked "How dangerous do you think the electromagnetic fields from mobile phones are while you talk on the phone, as illustrated in this picture?” and "How dangerous do you think the electromagnetic fields are from Wi-Fi routers in close proximity, as illustrated in this picture?” rated on a 7-point Likert scale $(1=$ not dangerous at all, 7 = very dangerous). The RPQ score was defined as the mean score from all responses.

\subsubsection{State and Trait Anxiety Index (STAI)}

The 40 item version of the STAI (Spielberger, Gorsuch, \& Lushene, 1970) was used to assess participant's state and trait anxiety. This comprises two, 20-item forms, assessing state (STAI-Y1) and trait (STAI-Y2) anxiety separately, with items answered on a 4-point Likert

scale $(1=$ not at all, $4=$ very much so $)$. Low, medium and high anxiety were defined as the as being less than minus 1 standard deviation from the mean, between minus 1 standard deviation and plus 1 standard deviation from the mean, and greater than plus 1 standard deviation from the mean respectively (Witthöft \& Rubin, 2013).

\subsubsection{NEO Five Factor Personality Index (NEO FFI)}

The 60 item NEO FFI (Costa \& McCrae, 1992) was used to assess personality traits: Extraversion, Agreeableness, Conscientiousness, Neuroticism, and Openness to Experience. This measure is beyond the scope of this paper and will not be discussed further. 


\subsection{Design}

In an experimental between-groups design, participants were assigned to the alarmist or control video group by a computerized random allocation process. In the alarmist video group, participants viewed a 3 min video appeal to the United Nations from a concerned scientist asking that more precautionary action be taken in regard to EMF exposure "from our favorite gadgets.” This included statements about the potential health risks from mobile phone and Wi-Fi signals, as well as sensationalized images of exposure scenarios (Blank, 2015). In the control group, participants viewed a 3 min segment of a documentary on gravity (Cox, 2013). This video contained no health related content. To minimize the influence of experimenter bias, a researcher not involved in data collection (AD) was responsible for the randomization and administration of the videos.

For the provocation trials, a randomized, counterbalanced, cross-over design was employed. Each participant's symptoms and belief of exposure was tested under a series of 14 sham and active provocation trials. The first two trials were non-blinded, open-label trials (1 RF-OFF, 1 RF-ON), where both the participant and the researcher were aware of the exposure status. This was verified to the participant using a Nardalert S3 broadband monitor (Narda Safety Test Solutions, Hauppauge, NY). These trials were followed by a series of 12 double-blind, randomized, counterbalanced trials (6 sham, 6 RF-ON). Randomization and counterbalancing was achieved using Excel (randomization command), such that a sham and RF-ON condition were treated as a pair; the conditions for each pair were randomly allocated before assigning the next pair; and no more than three of the same pair-order were permitted.

\subsection{Procedure}

A participant information sheet was sent to people who responded to recruitment flyers. This informed participants that a small percentage of the population report being sensitive to EMF, 
described some of the symptoms reported by IEI-EMF sufferers and explained that although the scientific evidence has yet to establish a clear relationship between exposure and symptoms, news reports about the possible adverse health effects of RF exposure continue to focus on people who report these symptoms. The general aims of the study were also listed in the information sheet.

Following a telephone screening interview, suitable participants were booked in for one mutually convenient testing session starting at 09:00 am, which lasted approximately $5.5 \mathrm{~h}$. Upon arrival at the laboratory, participants provided informed written consent and were given a verbal briefing of the ensuing testing session. After being instructed to switch off and leave all electronic devices in a general area of the laboratory, participants were then seated comfortably inside a Faraday cage, where they completed the baseline (Time 1 (T1)) measures of the STAI and RPQ. After completing the T1 questionnaires, participants then watched one of the two videos (based on their randomly assigned group). To maximize attention, participants were instructed to pay attention to the video as they would be required to answer questions about the video as part of a memory test at the conclusion of the study (although no memory test was conducted). After watching the video, participants again completed the STAI and RPQ (Time 2 (T2)). The exposure device was then set up and the provocation trials commenced, beginning with the initial 2 open-label trials (1 RF-OFF, 1 RF-ON), followed by the 12 double-blind trials. Each of the provocation trials lasted 20 min, beginning with a 5 min baseline interval, followed by a 10 min exposure interval (RF-ON or RF-OFF/sham, depending on randomization and counterbalancing), and concluded with a 5 min rest interval before the onset of the next trial. In each trial, participants were required to complete the SESS 2.5 mins into the baseline interval and again 7 mins into the exposure interval. At the conclusion of the provocation trials, participants were led out of the Faraday cage and asked whether they had any questions or concerns about any aspect of the 
experiment. No participants reported any concern about the experiment. A 15 min break was given after the $8^{\text {th }}$ provocation trial.

\subsection{Statistical analyses}

Statistical analyses were performed with SPSS Statistics for Windows, Version 23.0 (IBM, Armonk, New York). Where normality tests and visual inspection of the data revealed violations to the assumption of normality, non-parametric tests were employed and the corresponding effect sizes $(E S)$ were calculated as $r$, (where $0.1=$ small, $0.3=$ medium and 0.5 = large (Cohen, 1988)). Where parametric tests were conducted, corresponding effect sizes were calculated as Cohen's $d$, (where $0.3=$ small, $0.5=$ medium and $0.8=$ large (Cohen, 1988)).

\subsubsection{Preliminary Analyses:}

Independent samples $t$ tests were used to compare pre-existing (T1) levels of state anxiety (STAI-Y1), trait anxiety (STAI-Y2) and risk perception (RPQ) between the control and alarmist video groups. A Wilcoxon Signed-Ranks test was used to determine whether participants understood the exposure protocol by assessing whether there was a difference in belief of exposure rating of the SESS between the RF-OFF and RF-ON open-label trials.

\subsubsection{Hypothesis Driven Analyses:}

A Wilcoxon Signed-Ranks test was used to determine whether there was a main effect of exposure on the symptom score of the SESS in the open-label trials. To assess whether there was an interaction between video group and symptom score, a symptom difference score (RF-ON minus RF-OFF) was calculated and a Mann-Whitney U test used to compare the difference score between control and alarmist video groups in the open-label trials.

\subsubsection{Exploratory Analyses:}

To verify whether there was no effect of RF-EMF exposure, Wilcoxon Signed-Ranks tests were used to determine whether there was a main effect of exposure on either the belief of 
exposure rating or the symptom score of the SESS in the double blind trials. To assess whether there was an interaction between video group and either belief of exposure rating or symptom score, a difference score for each variable was calculated (RF-ON minus RF-OFF). These difference scores were calculated by averaging the belief of exposure, and separately the symptom difference scores (already calculated as the difference between the baseline and exposure intervals) of each variable across the 6 RF-ON and 6 RF-OFF conditions. The averaged RF-ON score was then subtracted from the averaged RF-OFF score. Mann-Whitney U tests were then used to compare each of these variables between the control and alarmist video groups.

Spearman's rho measure of association was used to test whether there was a relationship between belief of exposure and symptoms in the double-blind trials (irrespective of actual exposure condition) for each individual participant. The resultant rho values were then transformed using a Fisher transformation, and a one sample $t$ test was used to determine whether these transformed correlations differed from 0 . An independent samples $t$ test was used to assess whether there was a difference in the Fisher transformed Spearman's rho values between the control and alarmist video groups.

Mann-Whitney U tests were used to assess whether the difference between T1 and T2 STAIY1 score, and separately RPQ score, differed between the control and alarmist video groups. A Jonckheere-Terpstra test was used to determine whether there was a linear trend in the symptom score of the RF ON open-label trial as a function of pre-existing state anxiety.

\section{Results}

\subsection{Preliminary Analyses}

The means, standard deviations and test statistics for assessing whether there were significant differences between the control and alarmist video groups in relation to pre-existing levels of 
state anxiety (STAI Y-1), trait anxiety (STAI - Y2) and risk perception (RPQ) are displayed in Table 1. No significant differences were detected. Verifying that participants understood the exposure protocol (they believed that they were being exposed in the RF-ON condition and that they were not being exposed in the RF-OFF condition), belief of exposure ratings were significantly higher in the RF-ON (Median = 100) compared to the RF-OFF (Median = 0) condition, $T=0.00, z=-5.86$ (corrected for ties), $N$ - ties $=44, p<.001, E S=0.88$. All participants correctly reported that they were confident that the exposure was 'Definitely ON' in the RF-ON condition and 'Definitely OFF' in the RF-OFF condition of the open-label trials

Table 1: Descriptive statistics and tests for differences in pre-existing levels of state anxiety, trait anxiety and risk perception between the two video groups.

\begin{tabular}{cccc}
$\begin{array}{c}\text { Dependent } \\
\text { variable }\end{array}$ & $\begin{array}{c}\text { Control video } \\
\mathbf{N}=\mathbf{2 2}\end{array}$ & $\begin{array}{c}\text { Alarmist video } \\
\mathbf{N}=\mathbf{2 2}\end{array}$ & $\begin{array}{c}\text { Test statistic for } \\
\text { differences between } \\
\text { groups }\end{array}$ \\
\hline State Anxiety & $M=29.00, S D=8.11$ & $M=30.45, S D=9.96$ & $\begin{array}{c}t(42)=-0.639, \\
p=.527, E S=0.19\end{array}$ \\
Trait Anxiety & $M=38.45, S D=11.85$ & $M=36.41, S D=9.79$ & $t(42)=-0.624$, \\
& & & $p=.536, E S=0.19$ \\
Risk Perception & $M=2.35, S D=1.12$ & $M=2.51, S D=1.09$ & $t(42)=-0.477$, \\
& & & $p=.636, E S=0.14$ \\
\hline M = mean; SD = standard deviation; ES = effect size.
\end{tabular}

\subsection{Hypothesis Driven Analyses:}

\subsubsection{Symptoms in the open-label trials:}

Figure 1 shows the SESS symptom scores for the RF-OFF and RF-ON open-label trials. Overall, participants had significantly higher increases in symptom scores in the RF-ON 
condition $($ Median $=17.00)$ compared to the RF-OFF condition $($ Median $=-0.50), T=77.00$, $z=-4.476$ (corrected for ties), $N$ - ties $=40, p<.001, E S=0.71$.

\subsubsection{Effect of video group on symptoms in the open-label trials:}

The symptom scores in the RF-OFF condition were equal between the alarmist (Mean = 11.59 Median $=-3)$ and control $($ Mean $=11.45$ Median $=0)$ video groups, validating the comparison of symptom difference scores between the two groups. Figure 2 shows the symptom difference score (RF-ON - RF-OFF) for the control and alarmist video groups in the open-label trials. The symptom difference score was higher in the alarmist (Median = 25.50) compared to the control $($ Median $=5.00)$ video group, and the interaction between symptom difference score and video group was significant, $U=159.50, z=-1.738, p=.041$ (one-tailed), $E S=0.26^{1}$.

\footnotetext{
${ }^{1}$ One significant outlier was removed from this analysis. The interaction between symptom difference score and video group only reached trend level when including this outlier, $U=181.50, z=-1.421, p=.078$ (one-tailed), $E S=0.24$.
} 
Figure 1: The mean (dot), median (line), interquartile range (box) and range (whiskers) of the SESS symptom scores are shown for the RF-OFF and RF-ON open-label trials.

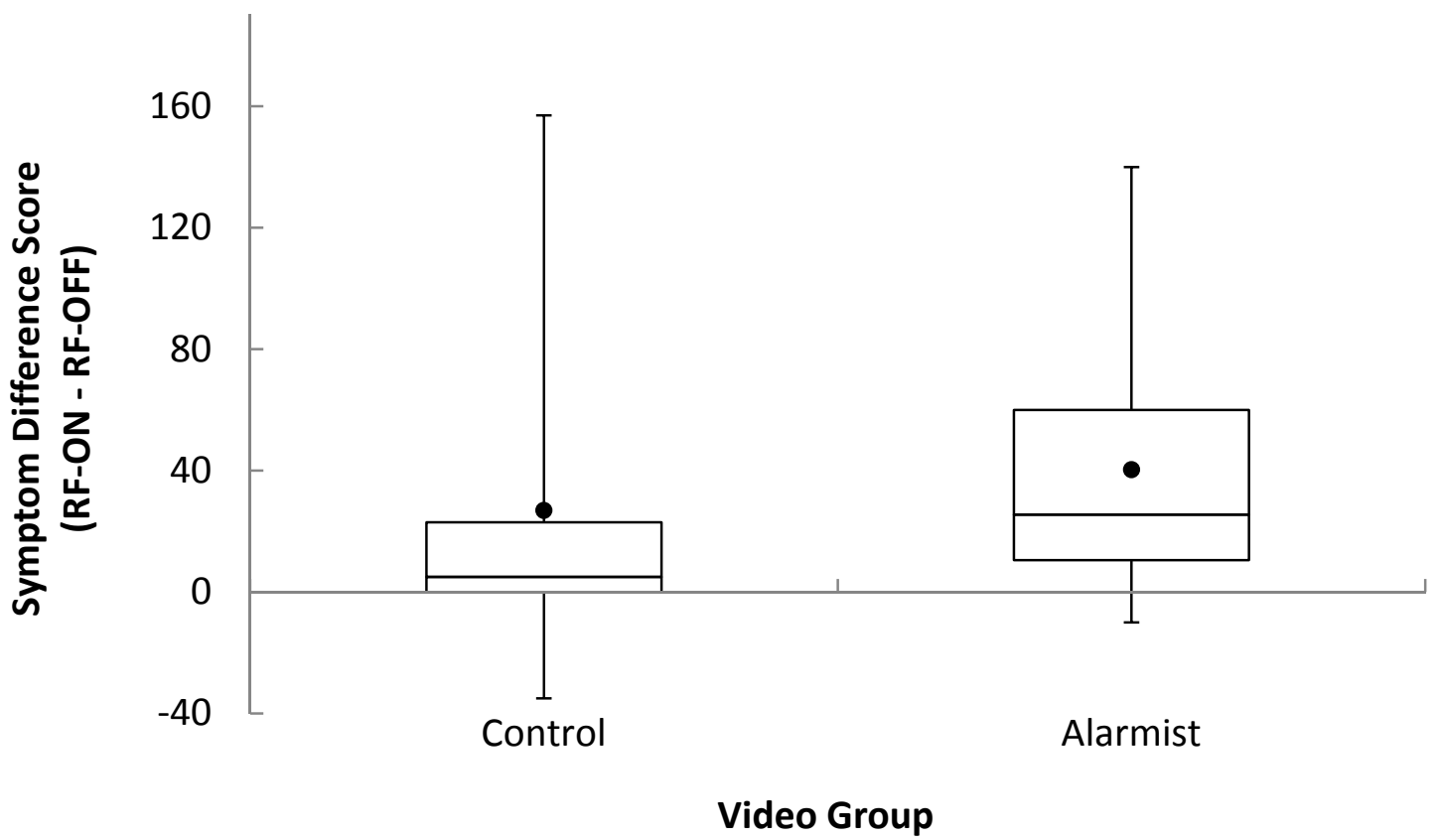

Figure 2: The mean (dot), median (line), interquartile range (box) and range (whiskers) of the difference in SESS symptom score (RF-ON - RF-OFF) are shown as a function of video group in the open-label trials ${ }^{1}$. 


\subsection{Exploratory Analyses}

\subsubsection{Effect of exposure on belief of exposure and symptoms in the double-blind trials:}

The SESS belief of exposure ratings in the RF-ON and sham double-blind trials are shown in Figure 3. Overall, there was no difference in belief of exposure rating between the RF-ON $($ Median $=34.58)$ and Sham conditions $($ Median $=38.33), T=331.00, z=-1.062($ corrected for ties), $N$-ties $=40, p=.144$ (one-tailed), $E S=0.17$, indicating that there was no main effect of exposure on belief of exposure rating. Three participants correctly identified at greater than chance levels (within-subjects), when they were and were not being exposed in the double-blind trials, with 1 participant getting 75\% correct and 2 participants getting $83 \%$ correct. No other participants could correctly identify when they were being exposed. Given a chance level of $5 \%$ and that there were 44 participants, it would be expected that 2.2 participants would correctly identify the conditions by chance.

The SESS symptom scores in the RF-ON and sham double-blind trials are shown in Figure 4. Overall, there was no difference in symptom score between the RF-ON (Median = 10.33) and Sham conditions (Median $=10.33), T=398.00, z=-0.906$ (corrected for ties), $N-$ ties $=43$, $p=.183$ (one-tailed), $E S=0.14$, indicating that there was no main effect of exposure on symptoms.

Spearman's rho measure of association showed that the relationships between belief of exposure and symptoms in the double-blind trials were highly variable between participants. These values ranged from -.276 to .882 in the control video group and -.675 to .852 in the alarmist video group. Following a Fisher transformation, a one-sample $t$ test found that the transformed rho values were significantly greater than $0, t(43)=6.862, p<.001, E S=1.03$. 


\subsubsection{Effect of video group on belief of exposure and symptoms in the double-blind trials:}

The belief of exposure difference score (RF-ON - Sham) also not differ between the control $($ Median $=-0.33)$ and alarmist $($ Median $=4.25)$ video groups, $U=186.50, z=-1.303, p=$ .096 (one-tailed), $E S=0.20$, indicating that there was no interaction between video group and belief of exposure rating. The symptom difference score (RF-ON - Sham) also did not differ between the control $($ Median $=-1.25)$ and alarmist $($ Median $=1.92)$ video groups, $U=$ 218.00, $z=-0.563, p=.287$ (one-tailed), $E S=0.08$, indicating that there was no interaction between video group and symptoms. 


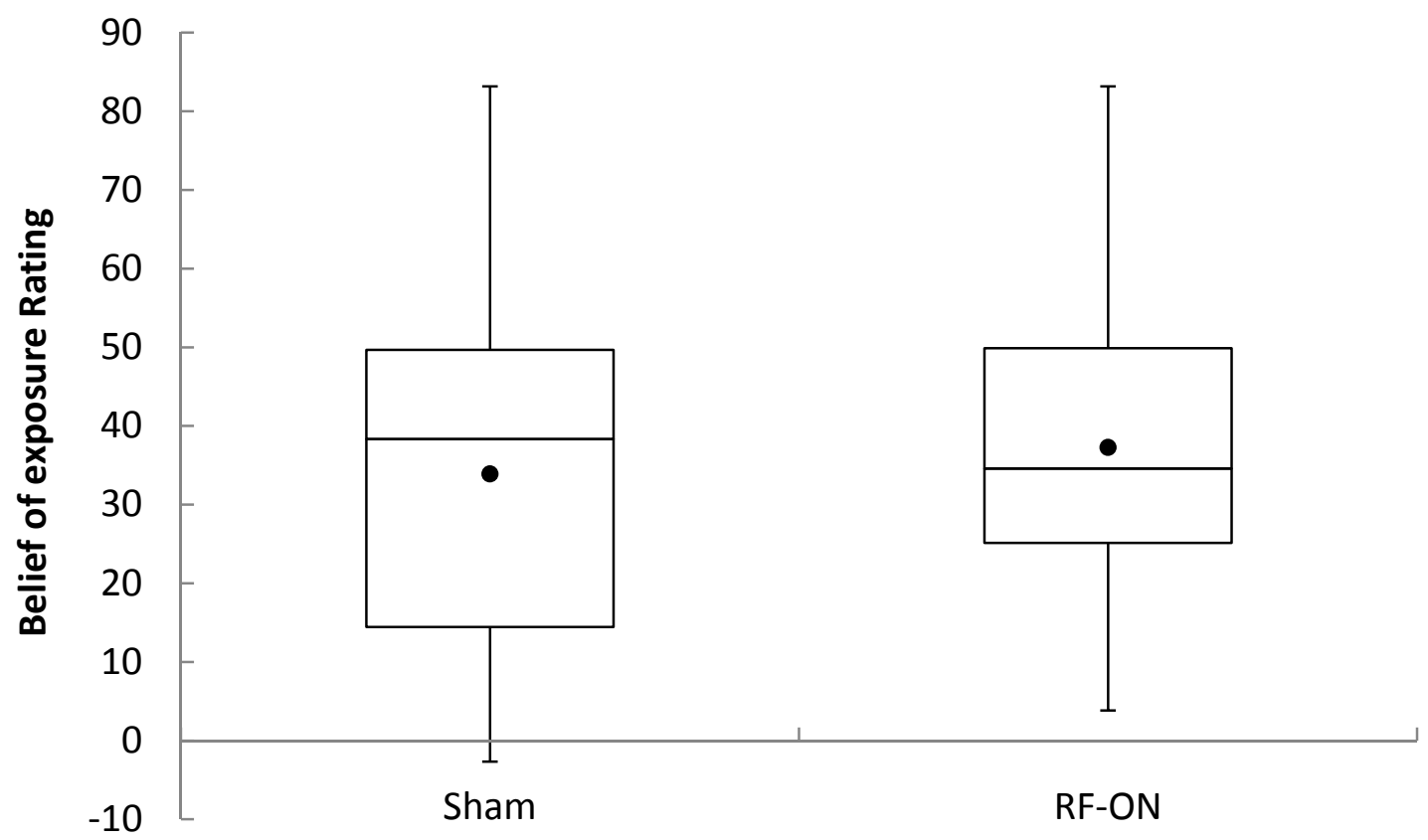

Exposure Condition

Figure 3: The mean (dot), median (line), interquartile range (box) and range (whiskers) of the SESS belief of exposure rating are shown for the sham and RF-ON double-blind trials.

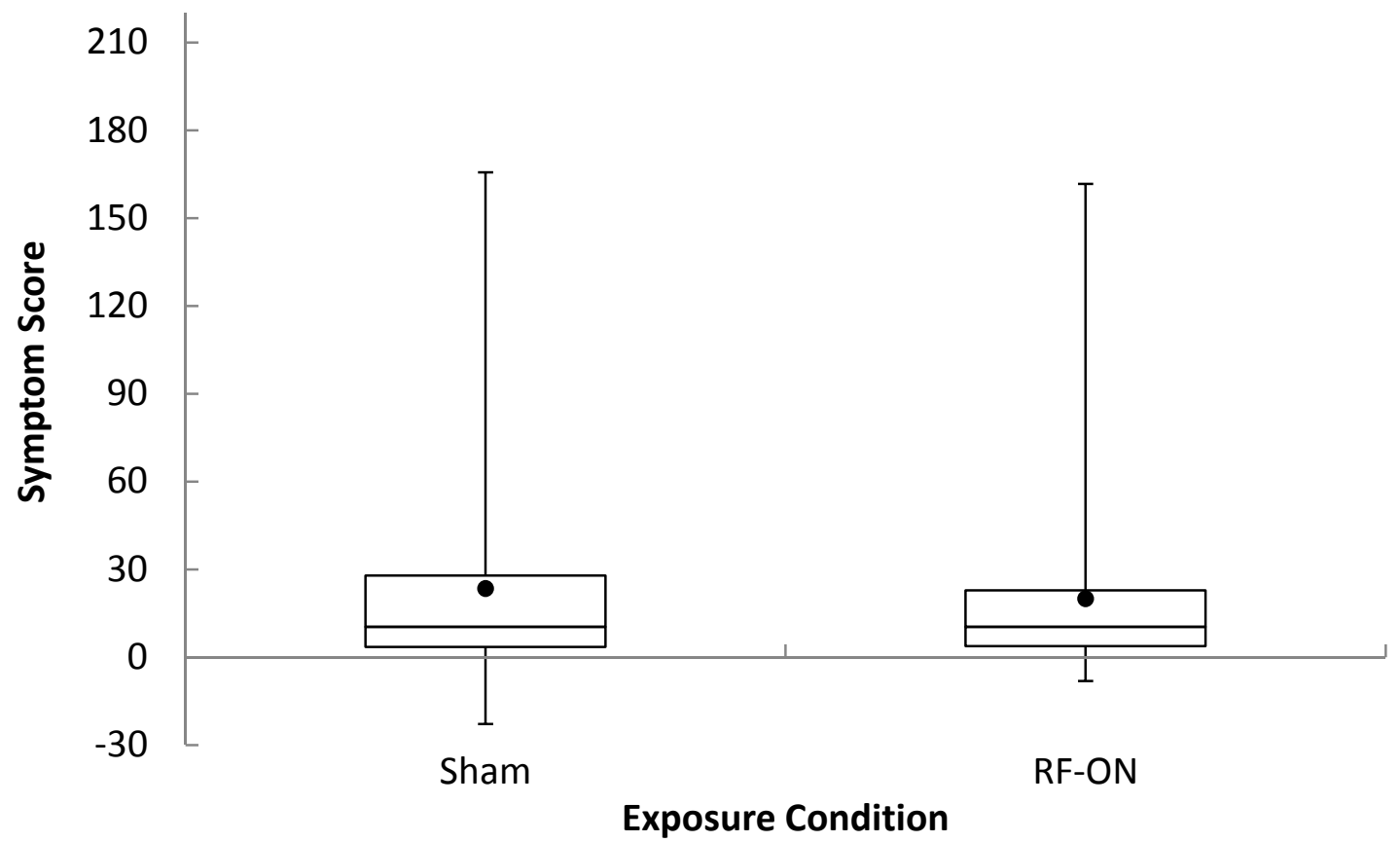

Figure 4: The mean (dot), median (line), interquartile range (box) and range (whiskers) of the SESS symptom score are shown for the sham and RF-ON double-blind trials. 


\subsubsection{Effect of video on state anxiety and risk perception:}

The difference in state anxiety (STAI-Y1) from T1 to T2 was significantly higher in the alarmist (Median $=3.50)$ compared to the control $($ Median $=-.50)$ video group, $U=135.50, z$ $=-2.505, p<.01$ (one-tailed), $E S=0.38$. The difference in risk perception (RPQ) from $\mathrm{T} 1$ to T2 was also significantly higher in the alarmist $($ Median $=1.00)$ compared to the control (Median $=0.00)$ video group, $U=75.50, z=-3.946, p<.001$ (one-tailed), $E S=0.60$.

\subsubsection{Relationship between belief of exposure and symptoms in double-blind trials as a}

\section{function of video group:}

The relationship between belief of exposure and symptoms (Fisher transformed Spearman's rho values $)$ did not differ between the control $(M=.47, S D=.48)$ and alarmist $(M=.54, S D$ $=.50)$ video groups, $t(42)=-.443, p=.660, E S=0.07$.

\subsubsection{Relationship between pre-existing state anxiety and symptoms in open-label trials:}

Figure 5 shows the SESS symptom scores as a function of anxiety group. No significant trend between symptom scores and higher levels of pre-existing anxiety was detected, $J=210.00, z$ $=-.838, p=.402, E S=0.13$. 


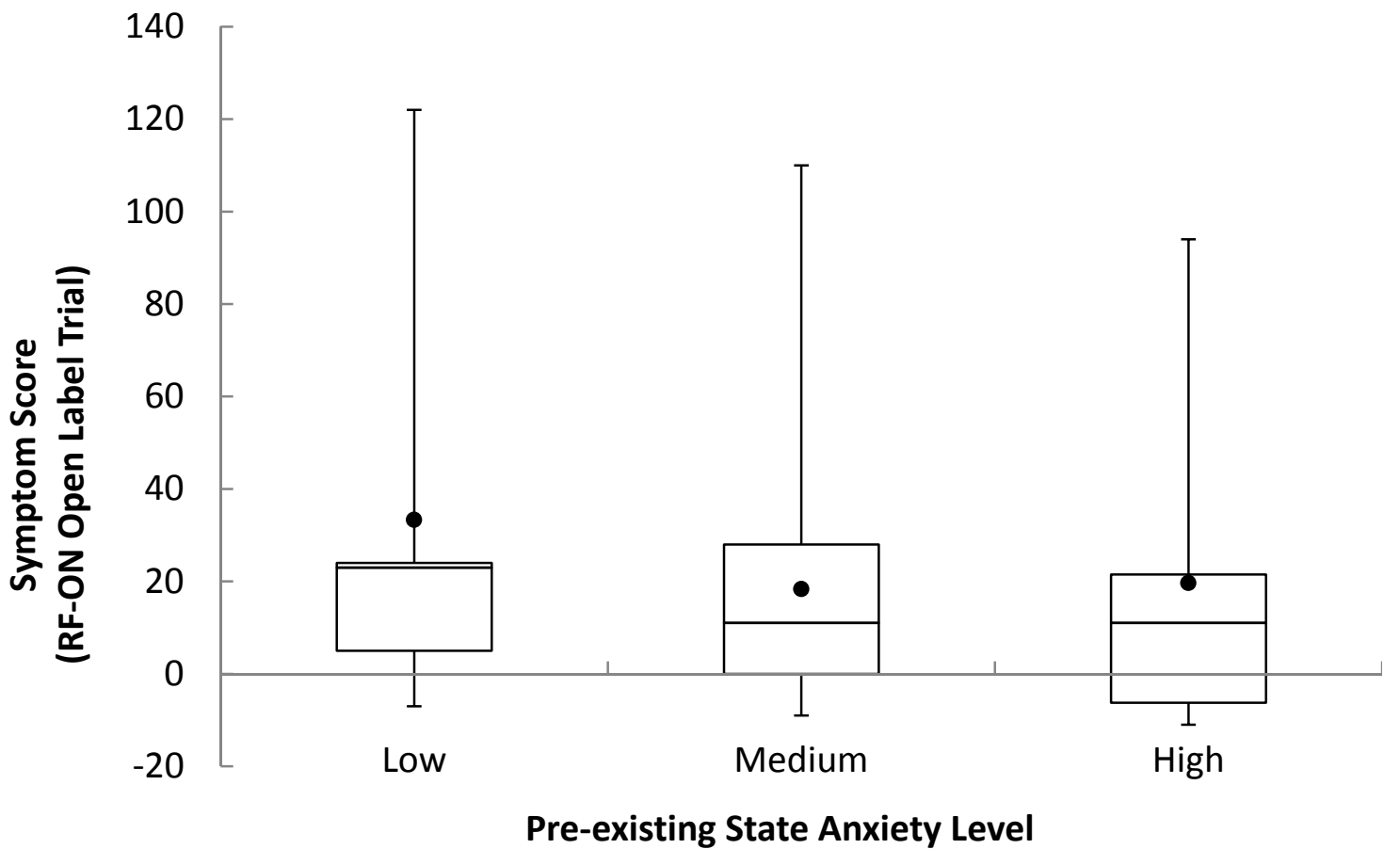

Figure 5: The mean (dot), median (line), interquartile range (box) and range (whiskers) of the SESS symptom scores in the RF-ON open-label trial are shown as a function of pre-existing state anxiety level.

\section{Discussion}

While there has been growing evidence that the symptoms reported by IEI-EMF sufferers are likely the result of a nocebo effect (Eltiti, Wallace, Ridgewell, et al., 2007; Nam et al., 2009; Oftedal et al., 2007; van Moorselaar et al., 2017; Verrender et al., 2018; Wilén et al., 2006), there has been limited understanding of the factors which contribute to such a response. Although a number of studies have shown that explicit suggestions about the adverse effects of EMF exposure can increase concern and negatively influence people's beliefs about EMF exposure (Barnett et al., 2007; Köteles et al., 2016; Nielsen et al., 2010; Wiedemann et al., 2014; Wiedemann et al., 2013; Wiedemann \& Schütz, 2005; Wiedemann et al., 2006; Witthöft et al., 2017) (factors which are considered to be key in contributing to a nocebo response (Webster et al., 2016)), it has remained relatively unclear whether the negative 
beliefs induced by such communications can result in greater symptom formation following a perceived exposure to EMF. The purpose of this experiment was to investigate whether perceived EMF exposure would elicit symptoms in a healthy population and to assess whether messages that emphasize 'adverse health effects of EMF exposure' can induce a nocebo response, including for those without high pre-existing levels of state anxiety. In the provocation trials, both active and sham EMF exposures were first demonstrated to participants in an initial non-blinded, open-label trial, before a series of double-blind, randomized, counterbalanced trials were conducted. By demonstrating that the experiment contained a 'no exposure' condition, the present study was able to more clearly determine whether any observed increases in symptoms were the result of a nocebo effect.

A number of preliminary tests were first used to establish whether the experiment was valid and whether the experimental manipulation had worked. These checks demonstrated that there were no differences in pre-existing levels of state anxiety, trait anxiety and EMF risk perception between the alarmist and control video groups. Further, these tests verified that participants understood the exposure protocol, as they correctly indicated that they were being exposed in the RF-ON open-label trial and not being exposed in the RF-OFF openlabel trial.

The results of the provocation trials revealed the crucial role of awareness and belief in the presentation of symptoms during perceived exposure to EMF. In the open-label trials, participants reported higher symptom scores in the RF-ON trial compared to the RF-OFF trial. In the subsequent double-blind trials, however, there was no difference in either belief of exposure or symptom scores between the RF-ON and sham conditions. These findings demonstrate that knowledge and/or awareness of the exposure condition was essential for producing an effect on symptoms. In addition to this, belief of exposure was found to be positively associated with higher symptom scores in the double-blind trials, giving further 
indication that a nocebo effect, rather than EMF exposure itself, was responsible for the increase in symptoms. In regards to the effect of messages which emphasize the 'adverse health effects of EMF exposure', the present study found that participants who viewed the alarmist video had higher symptom scores in the open-label trials than participants who viewed the control video. While, in contrast to Witthöft and Rubin (2013), the present study found that the effect of the video on symptom score was not moderated by pre-existing levels of state anxiety, it is important to note that the effect of the video on symptom score in the present study was only trend level when including an outlier in the sample. This may indicate that the effect of the video was strongly influenced by the individuals in the study. This corresponds to the notion that both situational factors (such as viewing a particular media report) and dispositional factors (such as personality traits) interact to influence people’s worries about the potential health hazards of modern life, though further research is required to clarify the personality traits which may be involved in moderating this effect (Witthöft et al., 2017). It is important to note, however, that sample size may also play a considerable role in the interaction between the type of video viewed and symptom score, and a larger sample size would have increased the chance of identifying an effect of the video in the present study. In line with previous research, the present study also found that participants who viewed the alarmist video had a larger increase in state anxiety and risk perception from baseline relative to those who viewed the control video (Bräscher, Raymaekers, et al., 2017; Witthöft et al., 2017; Witthöft \& Rubin, 2013). This provides further support to the notion that sensationalized media reports are capable of increasing people’s concerns and worries about exposure to EMF. Nonetheless, it is also possible that pre-existing beliefs about the relative harmfulness of EMF exposure may have influenced the results of this study via a ceiling effect. Future studies could usefully address this issue by using pre-screening to 
allocate participants into "high” and "low” risk perception groups, within each of the control and alarmist video groups.

Overall, the results of the present study corroborate those of IEI-EMF provocation studies, and demonstrate that the belief of being exposed, rather than EMF exposure itself, is sufficient to trigger symptoms in healthy participants, including those without high preexisting levels of anxiety. This is supported by the fact that $77 \%$ of participants reported higher symptoms in the open-label RF-ON trial compared to the RF-OFF trial, while no difference in symptom score was detected in the double-blind trials. Although the size of the effect on symptoms in the open-label trials in the present study was not as large as the effect observed for IEI-EMF sufferers in a previous study (ES > 3.6)(Verrender et al., 2018), the effect observed in the present study was still quite large $(E S=0.71)$, and is larger than the traditionally used nomenclature of Cohen (1988) (who treats the largest category of effect size as $>.05$ ). This may indicate that the nocebo response displayed by IEI-EMF sufferers during a perceived exposure situation is a normal human response. In addition to this, the results of the present study not only support those of previous studies suggesting that sensationalist media reports about perceived environmental hazards can raise concerns and negative beliefs about EMF, but also demonstrate that such reports may be contributing to a symptomatic nocebo response. This is analogous to the conclusions reached by studies investigating whether media health warnings can influence symptom expectations after exposure to infrasound from wind turbines or chemical pollution (Crichton, Dodd, Schmid, Gamble, \& Petrie, 2014; Winters et al., 2003) and further emphasizes the importance of disseminating accurate scientific and health information in order to reduce the likelihood of symptomatic nocebo responses in the community more generally. 
A number of potential limitations should be considered when interpreting the results of the present study. First, as the sample was mainly comprised of a relatively young healthy population, the present study is unable to comment on whether similar effects of alarmist media would be observed in a more general population sample. While online advertisements were used in an attempt to attract greater interest in the study, future studies could address this issue by using local newspapers and radio stations to recruit more broadly from the community. Second, as the RF-OFF condition always preceded the RF-ON condition in the open-label trials, the possibility that part of the increase in symptoms in the open-label trials (independent of the media content) was due to the elapsed study time cannot be completely ruled out. However, it is important to note that participants in provocation studies generally do not report such large increases in symptoms as a function of time (Schmidt, Wolfs-Takens, Oosterlaan, \& van den Hout, 1994). In addition to this, it is possible that effects on risk perception and symptoms may be triggered by any message on EMF and health, irrespective of whether it is an alarmist or positive message. However, Crichton and Petrie (2015a) found that positively framed health information may reverse or dilute the effect of negative expectations formed by alarmist media in the context of infrasound exposure, which suggests that the frame of the message is important for symptom perceptions. Nevertheless, future studies could include a third 'positive' video group to address this issue. Finally, due to feasibility requirements, the present study was limited to assessing acute symptom responses to acute exposures and the experiment was conducted in a laboratory setting using a Faraday cage. It is important that these factors are taken into consideration when interpreting the results of the present study, as these conditions may not reflect typical everyday exposure scenarios.

Although the present study has provided further evidence that symptoms attributed to EMF exposure are likely the result of a nocebo response, one of the major difficulties in treating 
IEI-EMF is the stigma attached to the notion that the condition is a psychological illness. While cognitive-behavioral therapy has been shown to be efficacious in treating IEI-EMF (Rubin, Das Munshi, \& Wessely, 2006), simply telling sufferers that their symptoms do not have a toxicological cause is not reassuring and is unlikely to completely alleviate symptoms (Rief, Heitmüller, Reisberg, \& Rüddel, 2006). For instance, although van Moorselaar et al. (2017) found that providing individual feedback on the results of double-blind provocation studies reduced IEI-EMF participants certainty about responding to acute EMF exposures, the feedback did not materially change IEI-EMF sufferers perception of being sensitive to EMF in their everyday life. Likewise, Nieto-Hernandez, Rubin, Cleare, Weinman, and Wessely (2008) found that providing feedback to IEI-EMF sufferers about their ability to discriminate between active and sham exposures had no influence on subsequent symptom levels or perceived sensitivity to EMF. Explaining the nocebo response may, however, offer an alternative solution. Recently, Crichton and Petrie (2015b) found that participants who reported symptomatic experiences during infrasound exposure returned mood and symptom levels to baseline levels in a subsequent exposure after they had received an explanation of the nocebo response with supporting scientific evidence. Whether such an effect could be replicated in people who experience IEI-EMF, however, remains unclear. Explaining that the nocebo response experienced by IEI-EMF sufferers is a normal human response may also offer a useful approach for addressing the condition in the future. Nonetheless, it is conceivable that explaining psychological mechanisms to people who claim to experience IEI-EMF may be interpreted as offensive or lacking credibility. It may thus be more appropriate (and more accurate) to emphasize that although EMF has not been shown to cause symptoms, that this does not mean that IEI-EMF symptoms are necessarily due to the nocebo effect; they may also relate to an undiagnosed medical condition (Dieudonné, 2016) which would require attention from a medical professional. Discussing the potential etiology 
of symptoms with IEI-EMF sufferers is thus a difficult task, and one that requires further investigation.

\section{Acknowledgments:}

The authors wish to express their thanks to Naomi Mansell for her assistance with the data collection for this study. 


\section{References}

Baliatsas, C., Bolte, J., Yzermans, J., Kelfkens, G., Hooiveld, M., Lebret, E., \& van Kamp, I. (2015). Actual and perceived exposure to electromagnetic fields and non-specific physical symptoms: An epidemiological study based on self-reported data and electronic medical records. International Journal of Hygiene and Environmental Health, 218, 331-344. doi: http://dx.doi.org/10.1016/j.ijheh.2015.02.001

Baliatsas, C., Van Kamp, I., Lebret, E., \& Rubin, G. J. (2012). Idiopathic environmental intolerance attributed to electromagnetic fields (IEI-EMF): A systematic review of identifying criteria. BMC Public Health, 12, 643. doi: http://dx.doi.org/10.1186/1471-2458-12-643

Barnett, J., Timotijevic, L., Shepherd, R., \& Senior, V. (2007). Public responses to precautionary information from the Department of Health (UK) about possible health risks from mobile phones. Health Policy, 82, 240-250. doi: 10.1016/j.healthpol.2006.10.002

Benedetti, F., Lanotte, M., Lopiano, L., \& Colloca, L. (2007). When words are painful: Unraveling the mechanisms of the nocebo effect. Neuroscience, 147, 260-271. doi: 10.1016/j.neuroscience.2007.02.020

Blank, M. (2015). International Scientist Appeal on Risks of Electromagnetic Fields Presented to the United Nations. Available online: https://vimeo.com/123468632.

Blettner, M., Schlehofer, B., Breckenkamp, J., Kowall, B., Schmiedel, S., Reis, U., . . BergBeckhoff, G. (2009). Mobile phone base stations and adverse health effects: Phase 1 of a population-based, cross-sectional study in Germany. Occupational and Environmental Medicine, 66, 118-123.

Bräscher, A.-K., Kleinböhl, D., Hölzl, R., \& Becker, S. (2017). Differential classical conditioning of the nocebo effect: Increasing heat-pain perception without verbal suggestions. Frontiers in Psychology, 8. doi: 10.3389/fpsyg.2017.02163

Bräscher, A.-K., Raymaekers, K., Van den Bergh, O., \& Witthöft, M. (2017). Are media reports able to cause somatic symptoms attributed to WiFi radiation? An experimental test of the negative expectation hypothesis. Environmental Research, 156, 265-271. doi: 10.1016/j.envres.2017.03.040

Claassen, L., Smid, T., Woudenberg, F., \& Timmermans, D. R. M. (2012). Media coverage on electromagnetic fields and health: Content analysis of Dutch newspaper articles and websites. Health, Risk \& Society, 14, 681-696. doi: 10.1080/13698575.2012.716820

Cohen, J. (1988). Statistical power analysis for the behavioural sciences (2nd ed.). New York: Academic Press.

Costa, P. T., \& McCrae, R. R. (1992). Revised NEO personality inventory and NEO five-factor inventory professional manual. Odessa, FL: Psychological Assessment Resources.

Cox, B. (2013). Size Matters. Wonders of Life: British Broadcasting Corporation.

Crichton, F., Dodd, G., Schmid, G., Gamble, G., \& Petrie, K. J. (2014). Can expectations produce symptoms from infrasound associated with wind turbines? Health Psychology, 33, 360-364. doi: $10.1037 / \mathrm{a} 0031760$

Crichton, F., \& Petrie, K. J. (2015a). Accentuate the positive: Counteracting psychogenic responses to media health messages in the age of the Internet. Journal of Psychosomatic Research, 79, 185-189. doi: 10.1016/j.jpsychores.2015.04.014

Crichton, F., \& Petrie, K. J. (2015b). Health complaints and wind turbines: The efficacy of explaining the nocebo response to reduce symptom reporting. Environmental Research, 140, 449-455.

Dieudonné, M. (2016). Does electromagnetic hypersensitivity originate from nocebo responses? Indications from a qualitative study. Bioelectromagnetics, 37, 14-24. doi: 10.1002/bem.21937

Eldridge-Thomas, B., \& Rubin, G. J. (2013). Idiopathic environmental intolerance ttributed to electromagnetic fields: A content analysis of british newspaper reports. PLOS ONE, 8, e65713. doi: 10.1371/journal.pone.0065713

Eltiti, S., Wallace, D., Ridgewell, A., Zougkou, K., Russo, R., Sepulveda, F., . . Fox, E. (2007). Does short-term exposure to mobile phone base station signals increase symptoms in individuals who report sensitivity to electromagnetic fields? A double-blind randomized provocation study. Environmental Health Perspectives, 115, 1603-1608. 
Eltiti, S., Wallace, D., Zougkou, K., Russo, R., Rasor, P., Fox, E., \& Joseph, S. (2007). Development and evaluation of the electromagnetic hypersensitivity questionnaire. Bioelectromagnetics, 28, 137-151. doi: 10.1002/bem.20279

Faul, F., Erdfelder, E., \& Buchner, A. (2007). G*Power 3: A flexible statistical power analysis program for the social, behavioural, and biomedical sciences. Behaviour Research Methods, 39, 175-191.

Freudenstein, F., Wiedemann, P. M., \& Brown, T. W. C. (2015). Exposure perception as a key indicator of risk perception and acceptance of sources of radio frequecy electromagnetic fields. Journal of Environmental and Public Health, 2015, 198272-198271-198272-198279. doi: http://dx.doi.org/10.1155/2015/198272

Frost, K., Frank, E., \& Maibach, E. (1997). Relative risk in the news media: a quantification of misrepresentation. American Journal of Public Health, 87, 842-845. doi: 10.2105/ajph.87.5.842

Hahn, R. A. (1997). The nocebo phenomenon: Concept, evidence, and implications for public health. Preventive Medicine, 26, 607-611. doi: http://dx.doi.org/10.1006/pmed.1996.0124

Health Canada. (2015). Safety code 6: Limits of human exposure to radiofrequency electromagnetic energy in the frequency range from $3 \mathrm{kHz}$ to $300 \mathrm{GHz}$. Canada: Consumer and Clinical Radiation Protection Bureau.

Health Council of the Netherlands. (2009). Electromagnetic fields: Annual update 2008. The Hague: Health Council of the Netherlands.

Hillert, L., Berglind, N., Arnetz, B. B., \& Bellander, T. (2002). Prevalence of self-reported hypersensitivity to electric or magnetic fields in a population-based questionnaire survey. Scandinavian Journal of Work, Environment \& Health, 28, 33-41.

ICNIRP. (1998). Guidelines for limiting exposure to time-varying electric, magnetic, and electromagnetic fields (up to $300 \mathrm{GHz}$ ). Health Physics, 74, 494-522.

Köteles, F., Tarján, E., \& Berkes, T. (2016). Artificial concerns. Effects of a commercial advertisement on modern health worries and sympathetic activation. Mentálhigiéné és Pszichoszomatika, 17, 61-79. doi: 10.1556/0406.17.2016.1.4

Levallois, P., Neutra, R., Lee, G., \& Hristova, L. (2002). Study of self-reported hypersensitivity to electromagnetic fields in California. Environmental Health Perspectives, 110, 619-623.

Nam, K. C., Lee, J. H., Noh, H. W., Cha, E. J., Kim, N. H., \& Kim, D. W. (2009). Hypersensitivity to RF fields emitted from CDMA cellular phones: A provocation study. Bioelectromagnetics, 30, 641-650.

Nielsen, J. B., Elstein, A., Gyrd-Hansen, D., Kildemoes, H. W., Kristiansen, I. S., \& Stovring, H. (2010). Effects of alternative styles of risk information on EMF risk perception. Bioelectromagnetics, 31, 504-512. doi: 10.1002/bem.20586

Nieto-Hernandez, R., Rubin, G. J., Cleare, A., Weinman, J., \& Wessely, S. (2008). Can evidence change belief? Reported mobile phone sensitivity following individual feedback of an inability to discriminate active from sham signals. Journal of Psychosomatic Research, 65, 453-460. doi: 10.1016/j.jpsychores.2008.04.005

Oftedal, G., Straume, A., Johnsson, A., \& Stovner, L. J. (2007). Mobile phone headache: A double blind, sham-controlled provocation study. Cephalalgia: An International Journal Of Headache, 27, 447-455.

Petrie, K. J., Sivertsen, B., Hysing, M., Broadbent, E., Moss-Morris, R., Eriksen, H. R., \& Ursin, H. (2001). Thoroughly modern worries. The relationship of worries about modernity to reported symptoms, health and medical care utilization. Journal of Psychosomatic Research, 51, 395401. doi: 10.1016/S0022-3999(01)00219-7

Petrie, K. J., \& Wessely, S. (2002). Modern worries, new technology, and medicine : New technologies mean new health complaints. BMJ : British Medical Journal, 324, 690-691.

Rief, W., Heitmüller, A. M., Reisberg, K., \& Rüddel, H. (2006). Why reassurance fails in patients with unexplained symptoms: An experimental investigation of remembered probabilities. PLOS Medicine, 3, e269. doi: 10.1371/journal.pmed.0030269

Röösli, M., Frei, P., Mohler, E., \& Hug, K. (2010). Systematic review on the health effects of exposure to radiofrequency electromagnetic fields from mobile phone base stations. Bulletin of the World Health Organization, 88, 887-896. doi: 10.2471/BLT.09.071852 
Röösli, M., Moser, M., Baldinini, Y., Meier, M., \& Braun-Fahrländer, C. (2004). Symptoms of ill health ascribed to electromagnetic field exposure - A questionnaire survey. International Journal of Hygiene and Environmental Health, 207, 141-150. doi: 10.1078/1438-4639-00269

Rubin, G. J., Das Munshi, J., \& Wessely, S. (2005). Electromagnetic hypersensitivity: A systematic review of provocation studies. Psychosomatic Medicine, 67, 224-232. doi: 10.1097/01.psy.0000155664.13300.64

Rubin, G. J., Das Munshi, J., \& Wessely, S. (2006). A systematic review of treatments for electromagnetic hypersensitivity. Psychotherapy and Psychosomatics, 75, 12-18. doi: 10.1159/000089222

Rubin, G. J., Nieto-Hernandez, R., \& Wessely, S. (2010). Idiopathic environmental intolerance attributed to electromagnetic fields (formerly 'electromagnetic hypersensitivity'): An updated systematic review of provocation studies. Bioelectromagnetics, 31, 1-11.

Schmidt, A. J. M., Wolfs-Takens, D. J., Oosterlaan, J., \& van den Hout, M. A. (1994). Psychological mechanisms in hypochondriasis: Attention-induced physical symptoms without sensory stimulation. Psychotherapy and Psychosomatics, 61, 117-120.

Schreier, N., Huss, A., \& Röösli, M. (2006). The prevalence of symptoms attributed to electromagnetic field exposure: A cross-sectional representative survey in Switzerland. Sozial- und Praventivmedizin, 51, 202-209. doi: 10.1007/s00038-006-5061-2

Schröttner, J., \& Leitgeb, N. (2008). Sensitivity to electricity: Temporal changes in Austria. BMC Public Health, 8, 310-310. doi: 10.1186/1471-2458-8-310

Scientific Committee on Emerging and Newly Idenified Health Risks. (2015). Potential health effects of exposure to electromagnetic fields (EMF).

Spielberger, C. D., Gorsuch, R. L., \& Lushene, R. E. (1970). The state-trait anxiety inventory (test manual) Palo Alto, CA: Consulting Psychologists Press.

Staudenmayer, H., Binkley, K. E., Leznoff, A., \& Phillips, S. (2003). Idiopathic environmental intolerance: Part 1: A causation analysis applying Bradford Hill's criteria to the toxicogenic theory. Toxicological Reviews, 22, 235-246.

Szemerszky, R., Köteles, F., Lihi, R., \& Bárdos, G. (2010). Polluted places or polluted minds? An experimental sham-exposure study on background psychological factors of symptom formation in 'Idiophatic Environmental Intolerance attributed to electromagnetic fields'. International Journal of Hygiene and Environmental Health, 213, 387-394. doi: 10.1016/j.ijheh.2010.05.001

Tseng, M.-C. M., Lin, Y.-P., \& Cheng, T.-J. (2011). Prevalence and psychiatric comorbidity of selfreported electromagnetic field sensitivity in Taiwan: A population-based study. Journal of the Formosan Medical Association, 110, 634-641. doi: 10.1016/j.jfma.2011.08.005

van Moorselaar, I., Slottje, P., Heller, P., van Strien, R., Kromhout, H., Murbach, M., . . Huss, A. (2017). Effects of personalised exposure on self-rated electromagnetic hypersensitivity and sensibility - A double-blind randomised controlled trial. Environment International, 99, 255262. doi: https://doi.org/10.1016/j.envint.2016.11.031

Verrender, A., Loughran, S. P., Anderson, V., Hillert, L., Rubin, G. J., Oftedal, G., \& Croft, R. J. (2018). IEI-EMF provocation case studies: A novel approach to testing sensitive individuals. Bioelectromagnetics, 39, 132-143. doi: 10.1002/bem.22095

Vrijheid, M., Armstrong, B. K., Bédard, D., Brown, J., Deltour, I., Iavarone, I., . . Cardis, E. (2009). Recall bias in the assessment of exposure to mobile phones. Journal of Exposure Science \& Environmental Epidemiology, 19, 369-381. doi: 10.1038/jes.2008.27

Webster, R. K., Weinman, J., \& Rubin, G. J. (2016). A systematic review of factors that contribute to nocebo effects. Health Psychology, 35, 1334-1355. doi: 10.1037/hea0000416

Wiedemann, P. M., Boerner, F. U., \& Repacholi, M. H. (2014). Do people understand IARC's 2B categorization of RF fields from cell phones? Bioelectromagnetics, 35, 373-378. doi: 10.1002/bem.21851

Wiedemann, P. M., Schuetz, H., Boerner, F., Clauberg, M., Croft, R., Shukla, R., . . Barnett, J. (2013). When precaution creates misunderstandings: The unintended effects of precautionary information on perceived risks, the EMF case. Risk Analysis, 33, 1788-1801. doi:

10.1111/risa.12034 
Wiedemann, P. M., \& Schütz, H. (2005). The precautionary principle and risk perception:

Experimental studies in the EMF area. Environmental Health Perspectives, 113, 402-405. doi: 10.1289/ehp.7538

Wiedemann, P. M., Thalmann, A. T., Grutsch, M. A., \& Schütz, H. (2006). The impacts of precautionary measures and the disclosure of scientific uncertainty on EMF risk perception and trust. Journal of Risk Research, 9, 361-372. doi: 10.1080/13669870600802111

Wientjes, C. J., \& Grossman, P. (1994). Overreactivity of the psyche or the soma? Interindividual associations between psychosomatic symptoms, anxiety, heart rate, and end-tidal partial carbon dioxide pressure. Psychosomatic Medicine, 56, 533-540.

Wilén, J., Johansson, A., Sandström, M., Kalezic, N., \& Lyskov, E. (2006). Psychophysiological tests and provocation of subjects with mobile phone related symptoms. Bioelectromagnetics, 27, 204-214. doi: 10.1002/bem.20195

Winters, W., Devriese, S., Van Diest, I., Nemery, B., Veulemans, H., Eelen, P., . . . Van den Bergh, O. (2003). Media warnings about environmental pollution facilitate the acquisition of symptoms in response to chemical substances. Psychosomatic Medicine, 65, 332-338.

Witthöft, M., Freitag, I., Nussbaum, C., Brascher, A. K., Jasper, F., Bailer, J., \& Rubin, G. J. (2017). On the origin of worries about modern health hazards: Experimental evidence for a conjoint influence of media reports and personality traits. Psychology and Health, 1-20. doi: 10.1080/08870446.2017.1357814

Witthöft, M., \& Rubin, G. J. (2013). Are media warnings about the adverse health effects of modern life self-fulfilling? An experimental study on idiopathic environmental intolerance attributed to electromagnetic fields (IEI-EMF). Journal of Psychosomatic Research, 74, 206-212. doi: 10.1016/j.jpsychores.2012.12.002 